subsequently." However, in discussing the "practical points" of evipan anaesthesia they mention the fact that premedication with omnopon and scopolamine diminishes the reflex movements of the patient and the sneezing reflex is less liable to occur. This consistency seems to detract from the strength of their case for the use of sodium evipan in ophthalmology.

Johnstone (Brit. Med. Jl., April 13, 1935, p. 761), states that in his experience premedication caused more unsatisfactory incidents and complications than in cases where this was omitted.

Sneezing can be lessened by using cocaine instillations into the conjunctival sac and some observers allege thai muscular twitchings may be diminished and sometimes abolished by increasing the dose but all agree that there are technical difficulties such as jerking the needle out of the vein or failure to enter the vein on account of the uncontrollable and violent movements of the patient.

It would seem that in the uncertainty of action of sodium evipan lies a very real danger. The disasters that may attend any operations on the eye through sneezing, coughing, muscular twitchings and the rise of intra-ocular pressure that these would cause is obvious and at the present it seems unjustifiable to run such risks particularly for intra-ocular operations when such measures as a facial nerve block and the injection of Tenon's capsule with a local anaesthetic, and a suture through the superior rectus may render operation possible even in patients most difficult to control. 'The main disadvantages which have attended the use of any intravenous anaesthetic up to date are the relative loss of control of the patient as compared with local anaesthesia, the uncertainty of the drug's action in some individuals and in many instances the inability to rectify serious complications when these occur during or after operation.

In the case of sodium evipan it may be said that until its action can be controlled with greater precision and certainty its use as an anaesthetic in ophthalmic surgery is limited.

\title{
ALL-INDIA OPHTHALMOLOGICAL SOCIETY
}

\section{Fourth Conference}

THE above Society held its Fourth Conference at Madras on April 22, 23 and 24, 1935. 'The President Elect was Lt.-Col. R. E. Wright, Professor of Ophthalmology and Superintendent, Govt. Ophthalmic Hospital, Madras. On the first day, Dr. E. V. Srinivasan, on behalf of the Reception Committee, welcomed the 
members; the Secretary, Dr. G. Zachariah, next introduced Sir Mahomed Usman, Ex-Acting Governor of Madras, who opened the session with a short address which dealt with the history of the Govt. Ophthalmic Hospital, Madras, and its influence in the spread of Ophthalmology in India, particularly in the direction of post-graduate teaching of recent $\cdot$ years. Col. Wright then delivered the Presidential address. In the afternoon a number of papers were read on "Nutritional disorders of the eye." The discussion centred chiefly around keratomalacia or xerophthalmia (these terms being used by speakers as equivalent to the congeries of pathological changes resulting from a polydeficiency in which vitaminosis "A" appears to play a prominent part). This common condition familiar to workers in the East for many years, plays a prominent rôle as a cause of preventible blindness. Contrary to views expressed by recent workers in England and elsewhere, skin rashes were not considered more frequent in keratomalacia than in the general hospital population, with the exception of lichen pilaris. 'T he same applies to pyogenic infections in general. The view was expressed that many laboratory workers were unfamiliar with the well developed human disorder as it occurs in bulk; hence certain divergencies of opinion in recent literature.

On the second day, the sessions were held in the Elliot Schoof theatre of the Govt. Ophthalmic Hospital. An interesting pro gramme of twelve short papers was given by members of the hospital staff on :--Sympathetic ophthalmitis, Occlusion of the central artery, White rings of the cornea, Corneal opacities due to sugar of lead, Corneal ulcer due to Glenospora graphii, Simple xerosis conjunctivae, Tuberculosis conjunctivae, Leptothricosis conjunctivae, von Hippel's disease, Birth injury of the cornea, Clinical aspects of glaucoma, Modern methods of anaesthesia in ophthalmic practice, Rare complications of cataract extraction. The paper on Sympathetic ophthalmitis, read by Dr. K. Koman Nayar, showed that the Govt. Ophthalmic Hospital experience was that this condition was less frequent in Indians than in Europeans. Discussion showed that this was not the universal opinion, Dr. B. K. Narayana Rao (Mysore) was in opposition to the view. Members were enabled to examine the extensive collection of intra-cranial lesion radiographs, water colour pictures and stereo-photos of external diseases of the eye, and the various other exhibits of the museum. A number of clinical cases were demonstrated including a group of 20 patients showing the various manifestations of keratomalacia, patients with acne rosacea, von Hippel's disease, Groenouw's keratitis, xerodermia pigmentosa, thrombosis of the central vein, and two keratoconus cases, the last three by Dr. Narayana Rao. Other papers read were on the rôte of anaesthesia in ophthalmology and three cases of Groenouw's disease. Prevention of blindness was 
discussed at the end of the afternoon session following an exhibition of coloured posters and lantern slides prepared for the Association for the Prevention of Blindness, Bengal, by Lt.-Col. E. O'G. Kirwan, Red Cross posters designed in New Delhi, and local Madras posters were also exhibited.

The final session was held in the Library Hall of the Madras Medical College. Papers were presented on :-The importance of the study of the field of vision on the prognosis and treatment of epidemic dropsy glaucoma; detachment of the retina, (26 cases); ocular complications due to focal sepsis ; the effects of subconjunctival injections of guaiacol cacodylate in phlyctenular keratoconjunctivitis; asepsis of the conjunctival sac; some observations on slit-lamp microscopy; treatment of immature senile cataract; cataract extraction in Aligarh; blue sclerotics with congenital syphilis; buphthalmos and blue sclerotics; diphtheria of the conjunctiva; unilateral trachoma; a short history of incidence of glaucoma in Bengal; ophthalmoplegic migraine involving VI nerve. The subject of greatest interest was primary non-congestive glaucoma, the Calcutta members bringing forward experience in connection with the recent outbreaks of epidemic dropsy. The fact that in the excessively high tensions of epidemic dropsy glaucoma, field changes and disc changes might be absent for months elicited an interesting discussion. Decompression by sclerocorneal trephining was still employed extensively in Calcutta, hundreds of operations having been done in the last few months.

In connection with the Prevention of Blindness, it was considered by the Committee that the responsibility of governing authorities was insufficiently realized and that certain recommendations should be put before the various authorities in British India and the Indian States. These will appear in due course. About sixty members attended.

\section{ABSTRACTS}

\section{MISCELLANEOUS}

(1) Minchin, R. L. Haviland (Indian Military Hospital, Abbottabad). - Blood stained tears. Lancet, January 19, 1935.

(1) Minchin reports the following case. "A girl, $3 \frac{1}{2}$ years of age, child of a Gurkha rifleman, was brought to hospital with the history that for the last three months whenever she cried blood and not tears came from the eyes. The child was examined 